\title{
,men \\ 2D/1D MXene/MWCNT Hybrid Membrane-Based Evaporator for Solar Desalination
}

\author{
Yawei Yang *(D), Yan Han, Jianqiu Zhao and Wenxiu Que *
}

check for

updates

Citation: Yang, Y.; Han, Y.; Zhao, J.; Que, W. 2D/1D MXene/MWCNT

Hybrid Membrane-Based Evaporator for Solar Desalination. Materials 2022, 15, 929. https://doi.org/10.3390/ ma15030929

\section{Academic Editor: Polina P. Kuzhir}

Received: 28 December 2021

Accepted: 22 January 2022

Published: 25 January 2022

Publisher's Note: MDPI stays neutral with regard to jurisdictional claims in published maps and institutional affiliations.

Copyright: (C) 2022 by the authors. Licensee MDPI, Basel, Switzerland. This article is an open access article distributed under the terms and conditions of the Creative Commons Attribution (CC BY) license (https:// creativecommons.org/licenses/by/ $4.0 /)$

\author{
Electronic Materials Research Laboratory, Key Laboratory of the Ministry of Education, International Center for \\ Dielectric Research, Shaanxi Engineering Research Center of Advanced Energy Materials and Devices, School of \\ Electronic Science and Engineering, Xi'an Jiaotong University, Xi'an 710049, China; \\ hanyan0302@stu.xjtu.edu.cn (Y.H.); jqzhao1994@163.com (J.Z.) \\ * Correspondence: ywyang@xjtu.edu.cn (Y.Y.); wxque@xjtu.edu.cn (W.Q.)
}

\begin{abstract}
Solar vapor generation through evaporation using photothermal materials is a promising candidate for seawater desalination. The $\mathrm{Ti}_{3} \mathrm{C}_{2}$ MXene membrane has exhibited photothermal behavior in solar water evaporation. However, dense packed two-dimensional (2D) MXene membrane with high reflection loss and insufficient vapor escape channels limited its solar evaporation performance. In this work, one-dimensional (1D) multi-walled carbon nanotubes (MWCNT) were added into 2D $\mathrm{Ti}_{3} \mathrm{C}_{2}$ nanosheets as the holder to form a $2 \mathrm{D} / 1 \mathrm{D}$ hybrid photothermal membrane. Owing to the 2D/1D hybrid structure, more effective broadband solar absorption, water transportation and vapor escape were achieved.
\end{abstract}

Keywords: MXene; MWCNT; hybrid membrane; solar desalination

\section{Introduction}

Extracting freshwater from saline water sources, known as the desalination, is a common way to solve the freshwater shortage issue all over the world. Among various desalination technologies, vapor generation from seawater driven by solar energy has become one of the most promising strategies for clean water harvesting [1]. Solar vapor generation technology is not only for seawater desalination, but also can be used for wastewater treatment and electricity generation $[2,3]$.

Although several examples of photothermal membranes, including carbon-related materials [4,5], narrow-bandgap semiconductors [6-8] and plasmonic nanoparticles [9,10], have been investigated for solar desalination, two-dimensional (2D) $\mathrm{Ti}_{3} \mathrm{C}_{2}$ MXene nanosheets with broadband spectrum absorption and an 100\% internal light-to-heat conversion efficiency have drawn intensive attention in this area [11,12]. 2D photothermal membranes located at the water-air interface have exhibited vapor generation capability and low heat loss into the bulk water. However, the pristine 2D nanosheets membrane is dense stacked, leading to a high light reflection and limited pathway for water molecules passing through, which seriously hinders solar evaporation performance [13,14]. Various morphologies of $\mathrm{Ti}_{3} \mathrm{C}_{2}$ nanomaterials have been reported for improving solar desalination performance, including aerogel, foam, hydrogel, membrane, monoliths, porous and nanocomposite [15]. For $2 \mathrm{D} \mathrm{Ti}_{3} \mathrm{C}_{2}$ nanosheets-based photothermal membranes, zero-dimensional (0D) $\mathrm{Au}$ nanoparticles, $2 \mathrm{D} \mathrm{MoS}_{2}$ nanosheets and three-dimensional (3D) $\mathrm{Cu}_{3} \mathrm{BiS}_{3}$ nanoflowers were intercalated into $2 \mathrm{D} \mathrm{Ti}{ }_{3} \mathrm{C}_{2}$ layers to form $2 \mathrm{D} / 0 \mathrm{D} \mathrm{Ti}{ }_{3} \mathrm{C}_{2} / \mathrm{Au}$ [16], 2D/2D Ti ${ }_{3} \mathrm{C}_{2} / \mathrm{MoS}_{2}$ [17] and $2 \mathrm{D} / 3 \mathrm{D} \mathrm{Ti}_{3} \mathrm{C}_{2} / \mathrm{Cu}_{3} \mathrm{BiS}_{3}[18]$ composite membranes, respectively. Hence, photothermal membrane with rough surface and porous structure by intercalating various dimensional nanostructures has become a candidate for promoting water transportation and vapor escape. Adding one-dimensional (1D) multi-walled carbon nanotubes (MWCNT) with excellent hydrophilicity and thermal conductivity into the 2D membrane is also considered as an effective strategy for enhancing the porosity, light absorption and thermal conduction 
to the interfacial water. In this work, the 2D/1D MXene/MWCNT hybrid membrane was fabricated as the solar absorber for enhancing the vapor generation and seawater desalination performance.

\section{Materials and Methods}

\subsection{Materials}

Al (1-3 $\mu \mathrm{m}, 99.5 \%)$, TiC (2-4 $\mu \mathrm{m}, 99 \%)$ and $\mathrm{Ti}(\leq 48 \mu \mathrm{m}, 99.99 \%)$ powders were purchased from Aladdin. Hydrochloric acid $(\mathrm{HCl}, 36-38 \%)$, sulfuric acid $\left(\mathrm{H}_{2} \mathrm{SO}_{4}, 95-98 \%\right)$, nitric acid $\left(\mathrm{HNO}_{3}, 65-68 \%\right)$ and ethanol (99.7\%) were provided by Sinopharm Chemical. Multi-walled carbon nanotubes (MWCNT) were purchased from Beijing Deke Daojin Science and Technology Co., Ltd., Beijing, China. Hydrophilic mixed cellulose ester filter membrane $(0.22 \mu \mathrm{m}$ in pores size, $5 \mathrm{~cm}$ in diameter) was purchased from Shanghai Xinya Purification Equipment Co., Shanghai, China. All chemicals were used as received without further purification.

\subsection{Preparation of $\mathrm{Ti}_{3} \mathrm{C}_{2}$ MXenen Nanosheets Suspension}

The mixture of $\mathrm{TiC}, \mathrm{Al}$ and $\mathrm{Ti}$ powders with a molar ratio of 2.0:1.2:1.0 was ball-milled with ethyl alcohol for $4 \mathrm{~h}$ at $300 \mathrm{rpm}$, and dried in vacuum at $60^{\circ} \mathrm{C}$. $\mathrm{Ti}_{3} \mathrm{AlC}_{2}$ was prepared by annealing the mixture to $1350{ }^{\circ} \mathrm{C}$ for $2 \mathrm{~h}$ in Ar flow. The sintered product was grinded and sieved through a 400 mesh screen, so that the particle size was controlled $<38 \mu \mathrm{m}$. $\mathrm{Ti}_{3} \mathrm{C}_{2}$ nanosheets were prepared by etching away the $\mathrm{Al}$ layer of $\mathrm{Ti}_{3} \mathrm{AlC}_{2}$ with etching solution. The etching solution was obtained by slowly dissolving $2 \mathrm{~g} \mathrm{LiF}$ in $15 \mathrm{~mL} 9 \mathrm{M} \mathrm{HCl}$ under stirring for $5 \mathrm{~min}$. Then, $1 \mathrm{~g} \mathrm{Ti}_{3} \mathrm{AlC}_{2}$ powder was slowly added into the etching solution at $35^{\circ} \mathrm{C}$ under stirring for $24 \mathrm{~h}$. The product was washed with water and centrifuged at $3500 \mathrm{rpm}$ for $2 \mathrm{~min}$ for several times until $\mathrm{pH}>6$. After the final cycle, the $\mathrm{Ti}_{3} \mathrm{C}_{2}$ precipitate was dispersed in $200 \mathrm{~mL}$ water, followed by degassing and $\mathrm{N}_{2}$ bubbling for $1 \mathrm{~h}$. Finally, the suspension was centrifuged at $3500 \mathrm{rpm}$ for $30 \mathrm{~min}$ to remove the unetched ingredients, and the supernatant was the exfoliated monolayer or few-layer $\mathrm{Ti}_{3} \mathrm{C}_{2}$ nanosheets suspension.

\subsection{Preparation of MWCNT Suspension}

The original MWCNT was treated by acid to improve its hydrophilicity. First, $60 \mathrm{~mL}$ $\mathrm{H}_{2} \mathrm{SO}_{4}$ was slowly added into $20 \mathrm{~mL} \mathrm{HNO}_{3}$ under stirring. Then, $1 \mathrm{~g}$ original MWCNT was slowly added into the mixed acid, and the mixture was heated to $80{ }^{\circ} \mathrm{C}$ for $2 \mathrm{~h}$ under stirring. The mixture was centrifuged at 10,000 rpm for $10 \mathrm{~min}$, and the product was washed with water for several times. Finally, the acidified MWCNT was well-dispersed in water to form MWCNT suspension.

\subsection{Fabrication of the $\mathrm{Ti}_{3} \mathrm{C}_{2}-\mathrm{MWCNT}$ Membrane-Based Evaporator}

Various amount of MWCNT suspension (0, $20 \mathrm{wt} \%, 50 \mathrm{wt} \%$ and $80 \mathrm{wt} \%$ ) was added into $\mathrm{Ti}_{3} \mathrm{C}_{2}$ nanosheets suspension under stirring, and the total mass of the two components was fixed to $10 \mathrm{mg}$. After mixing uniformly, the mixed suspension was vacuum filtered on a hydrophilic mixed cellulose ester filter membrane with a diameter of $4 \mathrm{~cm}$, followed by drying at $40{ }^{\circ} \mathrm{C}$ to form a $\mathrm{Ti}_{3} \mathrm{C}_{2}$-MWCNT membrane (Figure 1a). The corresponding sequential $\mathrm{Ti}_{3} \mathrm{C}_{2}$-MWCNT membranes were recorded as $\mathrm{Ti}_{3} \mathrm{C}_{2}, \mathrm{~T}-20 \mathrm{C}, \mathrm{T}-50 \mathrm{C}$ and T-80C, respectively. The $\mathrm{Ti}_{3} \mathrm{C}_{2}$-MWCNT membrane-based evaporator was fabricated according to our previous work (Figure $1 \mathrm{~b}$ ) [14], consists of three parts: (i) a piece of $\mathrm{Ti}_{3} \mathrm{C}_{2}-\mathrm{MWCNT}$ membrane as the solar absorber and vapor generator, (ii) a polystyrene foam as the thermal insulator and floater and (iii) a non-woven fabric as the water transportation pathway by capillary effect. 
a

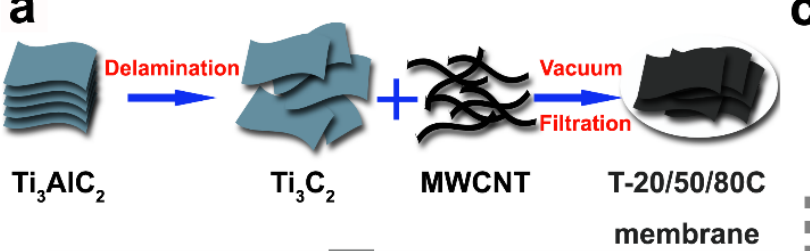

b

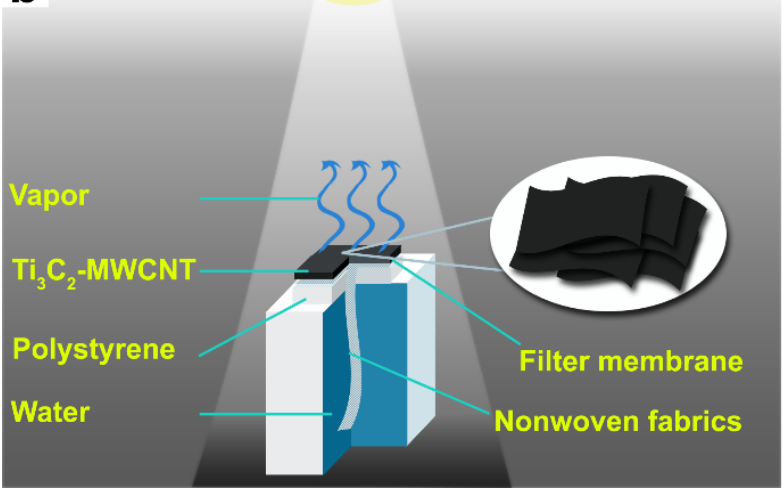

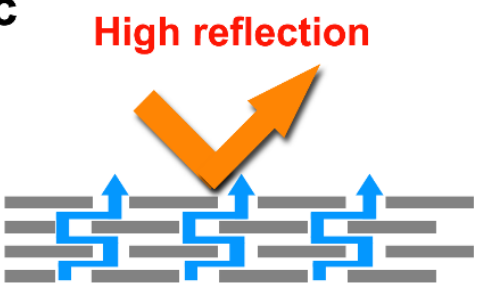

$\mathrm{Ti}_{3} \mathrm{C}_{2}$

Low reflection

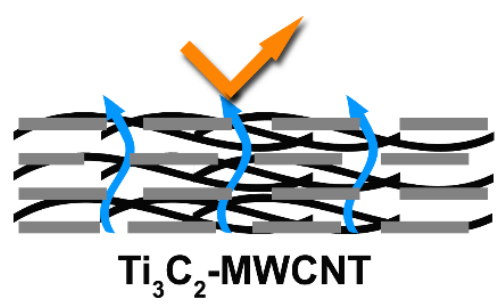

Figure 1. Scheme of (a) fabrication processes of the $\mathrm{Ti}_{3} \mathrm{C}_{2}$-MWCNT membranes, (b) the solar evaporator and (c) light reflection and vapor flux.

\subsection{Characterizations}

The crystal phase was analyzed by a powder X-ray diffraction spectroscopy (XRD, SmartLab, Rigaku, Akishima, Tokyo, Japan) with $\mathrm{Cu}$ Ka radiation $(40 \mathrm{kV}, 30 \mathrm{~mA})$. The microstructure was characterized by scanning electron microscopy (SEM, Quatan250 FEG, FEI, Hillsboro, OR, USA) and transmission electron microscopy (TEM, JEM-2100F, JEOL, Akishima, Tokyo, Japan). The UV-Vis-NIR absorption spectrum was measured by a UV/Vis/NIR spectrometer (PerkinElmer, Lambda 950, Waltham, MA, USA). A contact angle meter (JC2000D5, Powereach, Shanghai, China) was used to characterize the wettability. The salinity of water before and after solar evaporation was tracked by an inductively coupled plasma spectroscopy (ICP-OES, PerkinElmer Optima 8000, Waltham, MA, USA).

\subsection{Solar Vapor Generation Measurement}

The membrane was cut into a size of $2 \times 2 \mathrm{~cm}^{2}$. The evaporator was self-floated on the water in a Teflon container under the simulated solar illumination (Newport Oriel, $1.0 \mathrm{~kW} / \mathrm{m}^{2}$ ) at a temperature of $25 \sim 26{ }^{\circ} \mathrm{C}$ and a humidity of $30 \sim 40 \%$. The temperature was measured by an IR camera (Fluke, VT04A, Everett, WA, USA). The water weight loss through evaporation was measured by an electronic analytical balance $(0.1 \mathrm{mg}$ in accuracy). The corresponding solar-to-vapor conversion efficiency can be calculated by the evaporation rate derived from weight loss curve (see Supporting Information for details). The seawater desalination was performed by using natural seawater (Bohai Sea, salinity $\sim 2.75 \%)$.

\section{Results and Discussion}

The MWCNT and $\mathrm{Ti}_{3} \mathrm{C}_{2}$ nanosheets suspensions are stable homogeneous colloidal dispersions with particle size $<100 \mathrm{~nm}$ (Figures S1 and S2), which is benefit for membrane preparation. Two diffraction peaks at $2 \theta \approx 6^{\circ}$ and $26^{\circ}$ in XRD patterns can be indexed to (002) planes of $\mathrm{Ti}_{3} \mathrm{C}_{2}$ nanosheets and (002) planes of the graphite phase of MWCNT, respectively (Figure 2a). This result suggests that the $\mathrm{Ti}_{3} \mathrm{C}_{2}$ nanosheets remains $2 \mathrm{D}$ structure in the hybrid membrane. Besides, the hydrophilicity of the membrane has a positive impact on water transportation [19], and it improves with the increasing MWCNT content due to the adsorbed hydroxyl and carboxyl groups on MWCNT surface during acidification (Figure S3). The absorption of the $\mathrm{Ti}_{3} \mathrm{C}_{2}$-MWCNT hybrid membranes covers the entire solar spectrum region (Figure $2 \mathrm{~b}$ ). Two absorption peaks at $\sim 600 \mathrm{~nm}$ and $\sim 1000 \mathrm{~nm}$ can 
be assigned to the intrinsic absorption of the $2 \mathrm{D} \mathrm{Ti}_{3} \mathrm{C}_{2}$ membrane [14], which are almost covered by MWCNT absorption in T-80C due to the high MWCNT content. A strong light reflection can be seen in the $2 \mathrm{D} \mathrm{Ti}_{3} \mathrm{C}_{2}$ membrane due to its smooth surface (Figure $\mathrm{S} 4 \mathrm{a}, \mathrm{b}$ ). An increased light absorption is achieved for the hybrid membranes compared to the pristine $\mathrm{Ti}_{3} \mathrm{C}_{2}$ membrane, which should be attributed to the presence of MWCNT increasing light absorption and decreasing light reflection of the membrane (Figure S4c,d). As a result, the vapor temperature of all the hybrid membrane can rapidly reach to a steady high value of $\sim 38{ }^{\circ} \mathrm{C}$ within $4 \mathrm{~min}$ upon solar irradiation (Figure $2 \mathrm{c}$ ), which is due to the local heating of the membrane and a limited conduction loss of the thermal insulating layer. The vapor temperature rises a bit faster with the increasing MWCNT content in first 4 min. However, for the pristine $\mathrm{Ti}_{3} \mathrm{C}_{2}$ membrane, a lower steady vapor temperature of $\sim 36^{\circ} \mathrm{C}$ is achieved in a longer time of $6 \mathrm{~min}$.
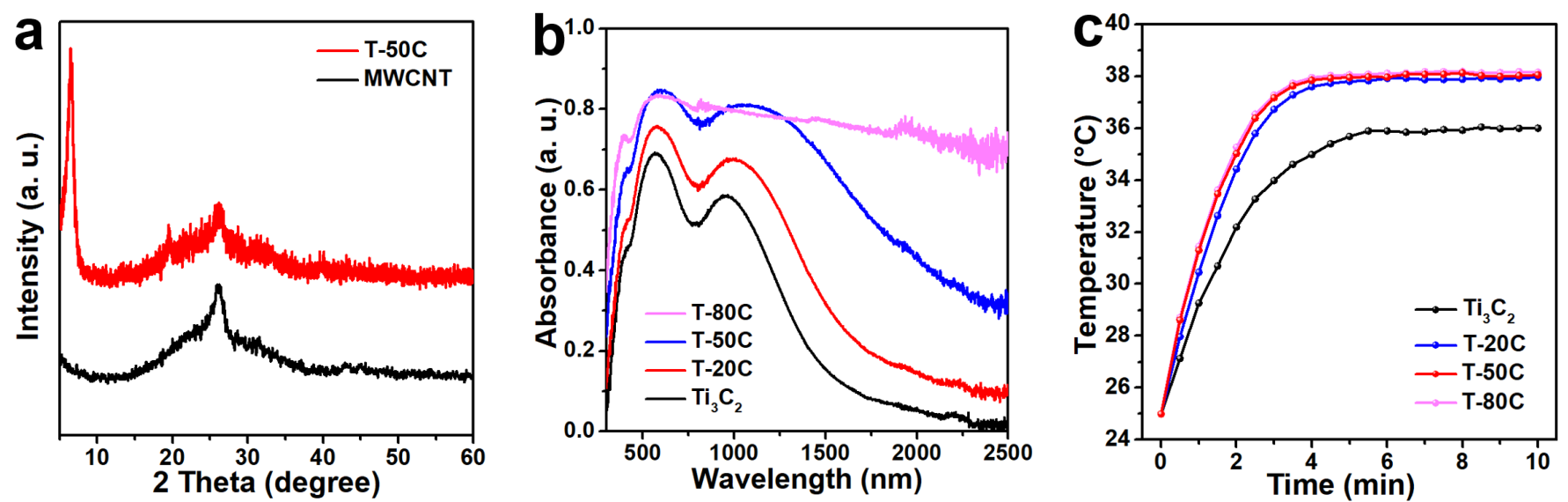

Figure 2. (a) XRD patterns, (b) UV-Vis-NIR absorption spectra and (c) temperature variation of the vapor over time of the $\mathrm{Ti}_{3} \mathrm{C}_{2}$-MWCNT membranes.

The $\mathrm{Ti}_{3} \mathrm{C}_{2}$ membrane exhibits a clear layered structure with a thickness of $\sim 3.9 \mu \mathrm{m}$ (Figure $3 a, b$ ) and a dense flat surface (Figure 3c), due to the compact stacking of the 2D nanosheets. For hybrid membranes, 1D MWCNT uniformly intercalates between the 2D $\mathrm{Ti}_{3} \mathrm{C}_{2}$ nanosheets as the holder, which enlarges the gaps between the layers, leading to a loose structure. As a result, with the increase of MWCNT content, the thickness of the hybrid membranes significantly increases to $\sim 7.7,7.2$ and $6.9 \mu \mathrm{m}$ for T-20C, T-50C and T-80C, respectively (Figure 3d and Figure S5a,d). The thicker composite layer indicates a loose structure of the membrane under the fixed total materials. The thickness of the $\mathrm{Ti}_{3} \mathrm{C}_{2}$-MWCNT hybrid membrane nearly doubles compared to pristine $\mathrm{Ti}_{3} \mathrm{C}_{2}$ membrane due to the interlaminated 1D MWCNT, but slightly decreases with the increase of MWCNT content because the layered structure fades away with the reducing nanosheets component, which compacts the membrane again (Figure $3 \mathrm{~d}$ and Figure S5a,d). The larger gaps between 2D layers (Figure 3b,e) and distinct pores in the surface (Figure 3f) formed by 1D MWCNT support inside the photothermal membrane promote the water transportation and vapor escape during solar evaporation [13].

The average evaporation rate of water, $\mathrm{Ti}_{3} \mathrm{C}_{2}, \mathrm{~T}-20 \mathrm{C}, \mathrm{T}-50 \mathrm{C}$ and T-80C membranes under one Sun irradiation is $0.42,1.41,1.47,1.55$ and $1.50 \mathrm{~kg} / \mathrm{m}^{2} \cdot \mathrm{h}$, respectively (Figure 4a). The optimized solar evaporation rate is 3.7 times higher than that of pristine water. The dark evaporation rate (natural volatilization) of all these membranes is $0.23 \mathrm{~kg} / \mathrm{m}^{2} \cdot \mathrm{h}$. Therefore, the net evaporation rate is $1.18,1.24,1.32$ and $1.27 \mathrm{~kg} / \mathrm{m}^{2} \cdot \mathrm{h}$, respectively. Hence, the average solar-to-vapor conversion efficiency $(\eta)$ is calculated by Equations (S1)-(S4) (Supporting Information) to be $80.7 \%, 85.3 \%, 90.8 \%$ and $87.3 \%$, respectively (Figure $4 \mathrm{~b}$ ), superior to most of the previously reported 2D materials-based photothermal membranes (Table S1). Obviously, when 1D MWCNT was introduced into the $2 \mathrm{D} \mathrm{Ti}{ }_{3} \mathrm{C}_{2}$ membrane, the evaporation rate and conversion efficiency of the hybrid membrane are significantly improved, which 
can be attributed to the enhanced light absorption and water molecules transportation of the $\mathrm{Ti}_{3} \mathrm{C}_{2}$-MWCNT hybrid membrane (Figure 1c). Although the light absorption ability of the hybrid membrane is improved with the increase of MWCNT content, the conversion efficiency increases firstly, and then decreases as the increase of MWCNT content. In other words, the T-50C membrane exhibits the highest solar evaporation performance, which indicates that the optimized 2D/1D structure is benefit for water transportation and vapor escape. It can be seen that there is an optimized membrane thickness for solar evaporation. Because too thin membrane cannot fully absorb the sunlight, but too thick one may loss heat during downward conduction. Therefore, there is a balance between the light absorption and heat conduction for the highest heat utilization. In the cycle running of the T-50C membrane with each cycle lasting for $12 \mathrm{~h}$, the solar evaporation rate is basically located in the range of $1.54 \sim 1.56 \mathrm{~kg} / \mathrm{m}^{2} \cdot \mathrm{h}$ with an average value of $1.55 \mathrm{~kg} / \mathrm{m}^{2} \cdot \mathrm{h}$ without decay (Figure 4c), suggesting the stability of the hybrid membrane. To evaluate the desalination performance of the membrane, the salinity before and after solar evaporation was tracked. The concentration of four primary ions $\left(\mathrm{Na}^{+}, \mathrm{K}^{+}, \mathrm{Mg}^{2+}\right.$ and $\left.\mathrm{Ca}^{2+}\right)$ is blocked over $99.5 \%$ (Figure $4 \mathrm{~d}$ ), outclassing the drinking standard. These results suggest that the $2 \mathrm{D} / 1 \mathrm{D} \mathrm{Ti} 3 \mathrm{C}_{2}$-MWCNT hybrid membrane-based evaporator is a good candidate for solar desalination.
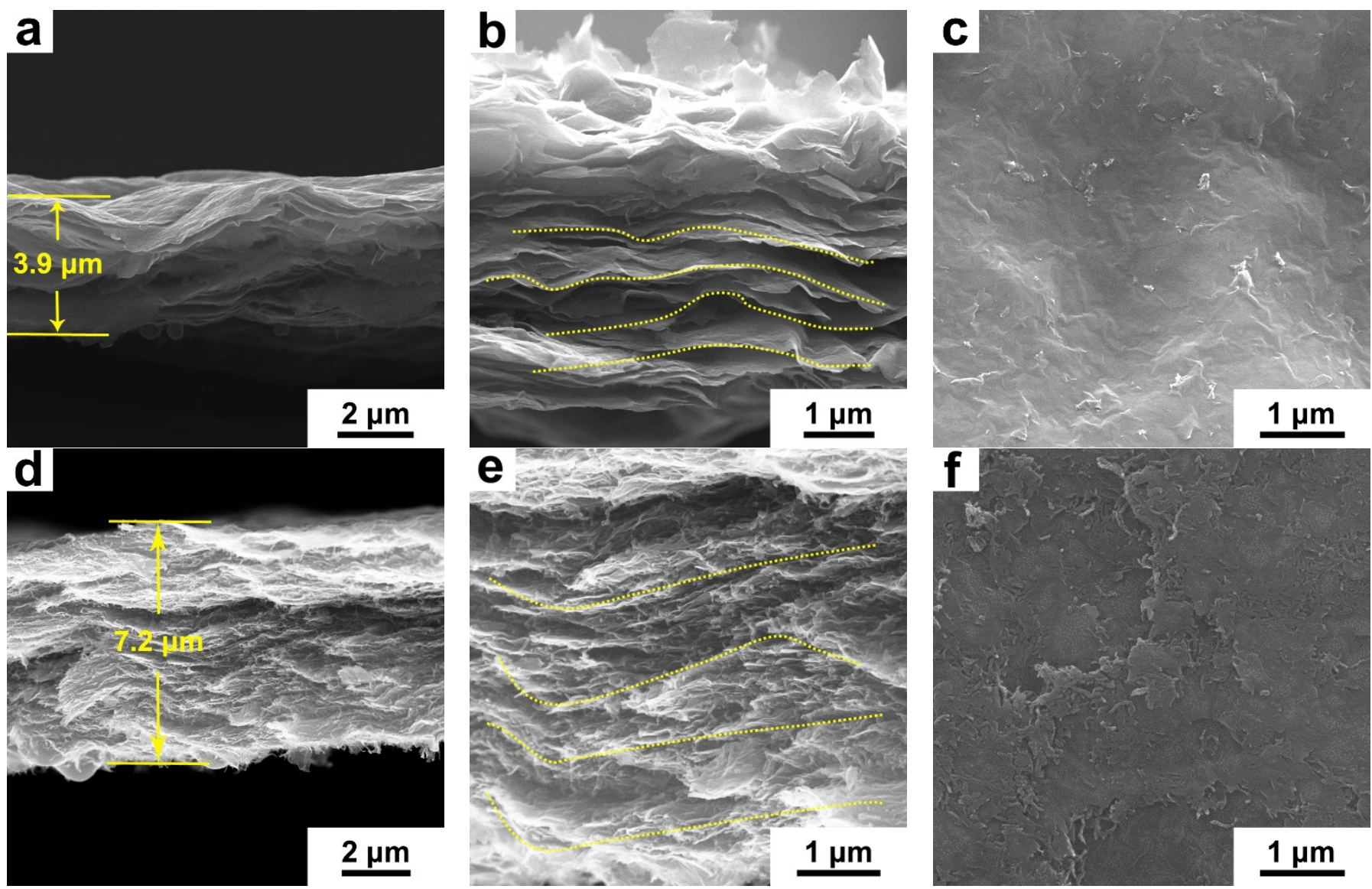

Figure 3. $(\mathbf{a}, \mathbf{b}, \mathbf{d}, \mathbf{e})$ Cross-section and $(\mathbf{c}, \mathbf{f})$ top-view $\mathrm{SEM}$ images of the $\mathrm{Ti}_{3} \mathrm{C}_{2} / \mathrm{T}-50 \mathrm{C}$ membranes. 

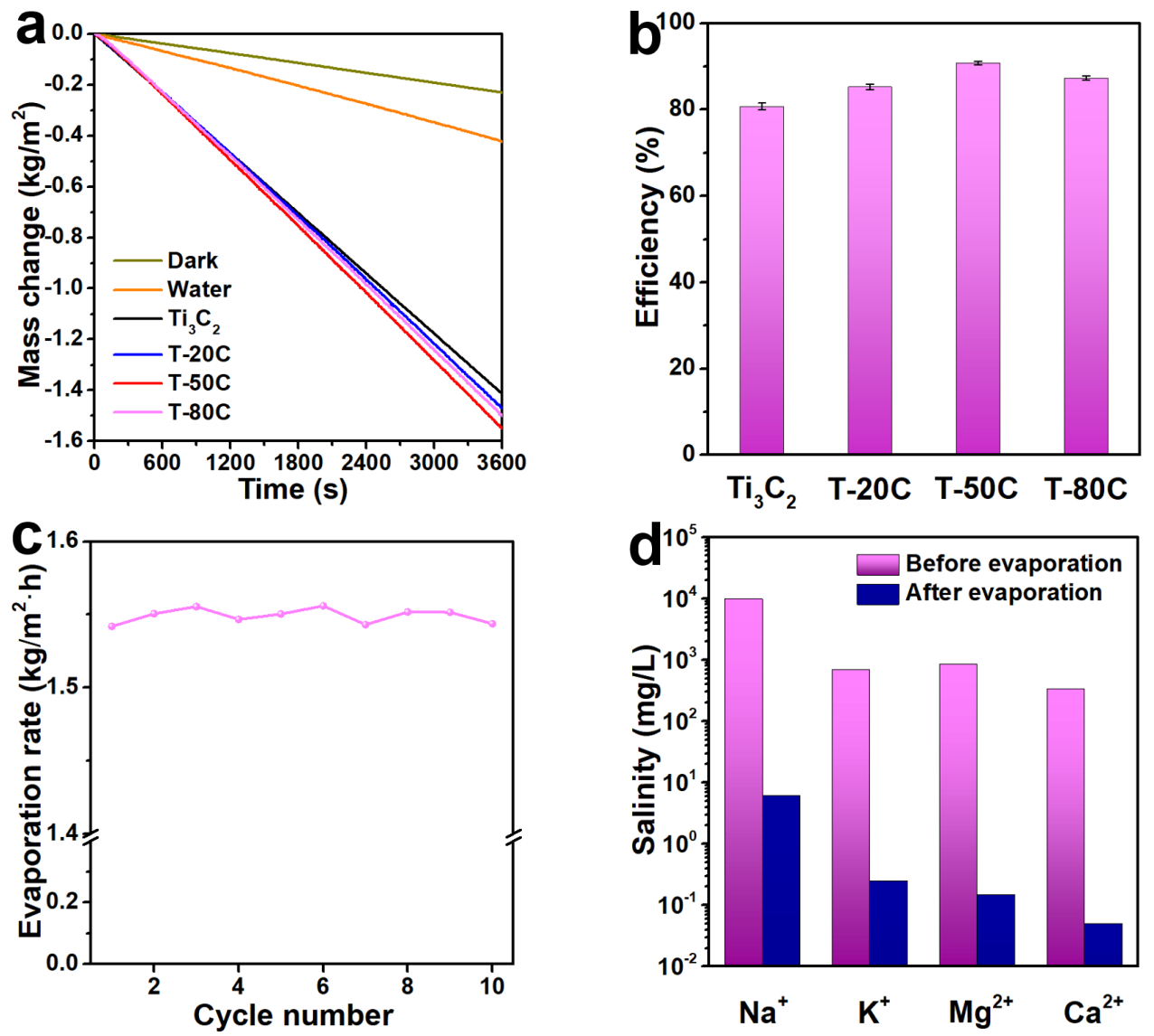

Figure 4. (a) Water weight loss through solar evaporation, (b) the corresponding solar-to-vapor conversion efficiency, (c) cycle running and (d) salinity before and after solar desalination.

\section{Conclusions}

1D MWCNT was added into 2D $\mathrm{Ti}_{3} \mathrm{C}_{2}$ nanosheets as the holder to form a hybrid photothermal membrane, which acts as the light absorber and vapor evaporator. An optimized solar evaporation rate of $1.55 \mathrm{~kg} / \mathrm{m}^{2} \cdot \mathrm{h}$, and corresponding solar-to-vapor conversion efficiency of $90.8 \%$ were achieved over the T-50C membrane, in which the light absorption, water transportation and vapor escape capabilities were significantly enhanced compared to the pristine $2 \mathrm{D} \mathrm{Ti}_{3} \mathrm{C}_{2}$ membrane. The present study is expected to provide a rational design for enhancing the solar vapor generation performance of the $2 \mathrm{D}$ membrane-based evaporators.

Supplementary Materials: The following supporting information can be downloaded at: https:/ / www.mdpi.com/article/10.3390/ma15030929/s1, Figure S1: Photographs of (a) the original MWCNT after $3 \mathrm{~h}$ of ultrasonic dispersion (left) and well-dispersed acidified MWCNT suspension (right), (b) no Tyndall effect in original MWCNT, (c) Tyndall effect in MWCNT suspension, and (d) Tyndall effect in $\mathrm{Ti}_{3} \mathrm{C}_{2}$ nanosheets suspension., Figure S2: TEM image of the acidified MWCNT., Figure S3: Contact angles of the $\mathrm{Ti}_{3} \mathrm{C}_{2}$-MWCNT membranes., Figure S4: Light reflection of the $(\mathrm{a}, \mathrm{b})$ pristine $\mathrm{Ti}_{3} \mathrm{C}_{2}$ and $(\mathrm{c}, \mathrm{d}) \mathrm{T}-50 \mathrm{C}$ membranes, Figure S5: $(\mathrm{a}, \mathrm{b}, \mathrm{d}, \mathrm{e})$ Cross-section and $(\mathrm{c}, \mathrm{f})$ top-view SEM images of the T-20C/T-80C membranes, Table S1: Solar desanlination performance of various 2D materials-based photothermal membranes.

Author Contributions: Conceptualization, Y.Y.; methodology, Y.Y.; investigation, Y.H. and J.Z.; data curation, J.Z.; writing—original draft preparation, Y.Y.; writing—review and editing, W.Q.; visualization, Y.H.; supervision, Y.Y. and W.Q.; funding acquisition, Y.Y. All authors have read and agreed to the published version of the manuscript.

Funding: This research was funded by the Natural Science Foundation of Shaanxi Province (No. 2021JQ-059), and Fundamental Research Funds for the Central Universities (No. xjh012020041). 
Institutional Review Board Statement: Not applicable.

Informed Consent Statement: Not applicable.

Data Availability Statement: The data supporting reported results of the current study are available from the corresponding authors on reasonable request.

Acknowledgments: The SEM work was conducted at International Center for Dielectric Research, Xi'an Jiaotong University.

Conflicts of Interest: The authors declare no conflict of interest.

\section{References}

1. Alvarez, P.J.J.; Chan, C.K.; Elimelech, M.; Halas, N.J.; Villagrán, D. Emerging opportunities for nanotechnology to enhance water security. Nat. Nanotechnol. 2018, 13, 634-641. [CrossRef] [PubMed]

2. Yang, Y.; Zhao, H.; Yin, Z.; Zhao, J.; Yin, X.; Li, N.; Yin, D.; Li, Y.; Lei, B.; Du, Y.; et al. A general salt-resistant hydrophilic/hydrophobicnanoporous double layer design for efficient andstable solar water evaporation distillation. Mater. Horiz. 2018, 5, 1143-1150. [CrossRef]

3. Hou, B.; Cui, Z.; Zhu, X.; Liu, X.; Wang, G.; Wang, J.; Mei, T.; Li, J.; Wang, X. Functionalized carbon materials for efficient solar steam and electricitygeneration. Mater. Chem. Phys. 2019, 222, 159-164. [CrossRef]

4. Zhang, P.; Li, J.; Lv, L.; Zhao, Y.; Qu, L. Vertically aligned graphene sheets membrane for highly efficient solar thermal generation of clean water. ACS Nano 2017, 11, 5087-5093. [CrossRef] [PubMed]

5. Liao, Y.; Chen, J.; Zhang, D.; Wang, X.; Yuan, B.; Deng, P.; Li, F.; Zhang, H. Lotus leaf as solar water evaporation devices. Mater. Lett. 2019, 240, 92-95. [CrossRef]

6. Yang, Y.; Que, W.; Zhao, J.; Han, Y.; Ju, M.; Yin, X. Membrane assembled from anti-fouling copper-zinc-tin-selenide nanocarambolas for solar-driven interfacial water evaporation. Chem. Eng. J. 2019, 373, 955-962. [CrossRef]

7. Liu, H.; Chen, C.; Wen, H.; Guo, R.; Williams, N.A.; Wang, B.; Chen, F.; Hu, L. Narrow bandgap semiconductor decorated wood membrane for high-efficiency solar-assisted water purification. J. Mater. Chem. A 2018, 6, 18839-18846. [CrossRef]

8. Ibrahim, I.; Seo, D.H.; McDonagh, A.M.; Shon, H.K.; Tijing, L. Semiconductor photothermal materials enabling efficient solar steamgeneration toward desalination and wastewater treatment. Desalination 2021, 500, 114853. [CrossRef]

9. Chen, J.; Feng, J.; Li, Z.; Xu, P.; Wang, X.; Yin, W.; Wang, M.; Ge, X.; Yin, Y. Space-confined seeded growth of black silver nanostructures for solar steam generation. Nano Lett. 2018, 19, 400-407. [CrossRef]

10. Yang, F.; Chen, J.; Ye, Z.; Ding, D.; Myung, N.V.; Yin, Y. Ni-based plasmonic/magnetic nanostructures as efficient light absorbers for steam generation. Adv. Funct. Mater. 2021, 31, 2006294. [CrossRef]

11. Li, R.; Zhang, L.; Shi, L.; Wang, P. MXene $\mathrm{Ti}_{3} \mathrm{C}_{2}$ : An effective 2D light-to-heat conversion material. ACS Nano 2017, 11, 3752-3759. [CrossRef] [PubMed]

12. Zhao, X.; Zha, X.J.; Pu, J.H.; Bai, L.; Bao, R.Y.; Liu, Z.Y.; Yang, M.B.; Yang, W. Macroporous three-dimensional MXene architectures for highly efficient solar steam generation. J. Mater. Chem. A 2019, 7, 10446-10455. [CrossRef]

13. Wang, Y.; Wang, C.; Song, X.; Megarajan, S.K.; Jiang, H. A facile nanocomposite strategy to fabricate a rGO-MWCNT photothermal layer for efficient water evaporation. J. Mater. Chem. A 2018, 6, 963-971. [CrossRef]

14. Zhao, J.; Yang, Y.; Yang, C.; Tian, Y.; Han, Y.; Liu, J.; Yin, X.; Que, W. A hydrophobic surface enabled salt-blocking 2D Ti ${ }_{3} \mathrm{C}_{2} \mathrm{MXene}$ membrane for efficient and stable solar desalination. J. Mater. Chem. A 2018, 6, 16196-16204. [CrossRef]

15. Sreedhar, A.; Noh, J. Advancements in solar desalination of seawater by various $\mathrm{Ti}_{3} \mathrm{C}_{2} \mathrm{MXene}$ based morphologies for freshwater generation: A review. Catalysts 2021, 11, 1435. [CrossRef]

16. Qu, W.; Zhao, H.; Zhang, Q.; Xia, D.; Tang, Z.; Chen, Q.; He, C.; Shu, D. Multifunctional Au/Ti ${ }_{3} \mathrm{C}_{2}$ photothermal membrane with antibacterial ability for stable and efficient solar water purification under the full spectrum. ACS Sustain. Chem. Eng. 2021, 9, 11372-11387. [CrossRef]

17. Xu, R.; Wei, N.; Li, Z.; Song, X.; Li, Q.; Sun, K.; Yang, E.; Gong, L.; Sui, Y.; Tian, J.; et al. Construction of hierarchical 2D/2D $\mathrm{Ti}_{3} \mathrm{C}_{2} / \mathrm{MoS}_{2}$ nanocomposites for high-efficiency solar steam generation. J. Colloid Interface Sci. 2021, 584, 125-133. [CrossRef] [PubMed]

18. Wang, Z.; Yu, K.; Gong, S.; Mao, H.; Huang, R.; Zhu, Z. $\mathrm{Cu}_{3} \mathrm{BiS}_{3} / \mathrm{MXenes}$ with excellent solar-thermal conversion for continuous and efficient seawater desalination. ACS Appl. Mater. Interfaces 2021, 13, 16246-16258. [CrossRef] [PubMed]

19. Ito, Y.; Tanabe, Y.; Han, J.; Fujita, T.; Tanigaki, K.; Chen, M. Multifunctional porous graphene for high-efficiency steam generation by heat localization. Adv. Mater. 2015, 27, 4302-4307. [CrossRef] [PubMed] 